\title{
Umbilical Endometriosis without Pelvic Surgery
}

\author{
Kenji Niwa ${ }^{1}$, Yusuke Mizuno ${ }^{2,3}$, Ryuichiro Yano4, Yoko Ueda1, Nozomi Narikawa, ${ }^{1,5}$, \\ Takuji Tanaka ${ }^{6}$ \\ ${ }^{1}$ Department of Obstetrics \& Gynecology, Gujo City Hospital, Gujo, Japan \\ ${ }^{2}$ Department of Surgery, Gujo City Hospital, Gujo, Japan \\ ${ }^{3}$ Department of General and Cardiothoracic Surgery, Gifu University Graduate School of Medicine, Gifu, \\ Japan \\ ${ }^{4}$ Department of Obstetrics \& Gynecology, Gifu University Graduate School of Medicine, Gifu, \\ Japan \\ ${ }^{5}$ Department of Obstetrics \& Gynecology, Japanese Red Cross Takayama Hospital, Takayama, \\ Japan \\ ${ }^{6}$ Department of Diagnostic Pathology (DDP) \& Research Center of Diagnostic Pathology (RC-DiP), Gifu \\ Municipal Hospital, Gifu, Japan \\ Email: kniwa.gujo913@gmail.com
}

Received 28 August 2014; revised 18 September 2014; accepted 30 September 2014

Copyright (C) 2014 by authors and Scientific Research Publishing Inc.

This work is licensed under the Creative Commons Attribution International License (CC BY).

http://creativecommons.org/licenses/by/4.0/

(c) (i) Open Access

\section{Abstract}

We report a recently observed case of primary umbilical endometriosis without previous pelvic surgery. A 41-year-old Japanese woman complained of umbilical nodular tumor. Histopathology revealed endometriosis of an approximate $10 \mathrm{~mm}$ resected mass. The stromal cells in endometriosis were immunohistochemically positive for CD10. Two months later the first umbilical surgery, she underwent a left salpingo-oophorectomy and release of adhesion around the left adnexa under a laparoscope. She was treated with dienogest $(2 \mathrm{mg} /$ day) for six months after four injections of GnRH analogue for four months. After three years of the follow-up, there were no signs of local relapse and no clinical and ultrasonographic abnormalities due to endometriosis.

\section{Keywords}

Umbilical Endometriosis, No Pelvic Surgery, Preservation of the Umbilicus, CD10, Immunohistochemistry

\footnotetext{
${ }^{*}$ Corresponding author.
} 


\section{Introduction}

Endometriosis is defined as the presence of endometrial glands and stroma abnormal located outside the uterine cavity. It is a benign gynecological disorder affecting $10 \%$ - 15\% of all women of reproductive age and presents an important cause of infertility [1] [2]. Common locations of endometriosis are pelvic organs, mostly the ovaries, fallopian tubes, utero-sacral ligaments, recto-vaginal septum and pelvic peritoneum. Although the different theories have been postulated to elucidate the pathogenesis of the endometriosis, none of them has been proven to be completely exhaustive to date [3] [4]. Clinical manifestations show the pelvic pain arising around menstration, menhorrage, painful intercourse, intestinal and urinary complaints [4] [5].

Extra-pelvic endometriosis has been described in almost every organ (i.e. gastro-intestinal organ, skin, diaphragm, lung and brain) [2]-[5]. Umbilical endometriosis (UE) is also known as Villar's nodule, named after the physician who first described the disease in 1886 [6]. While UE usually occurs secondary to surgical scars [7] [8], primary UE is extremely rare [4] [6] [9] [10]. We report here our recently observed case of UE without a previous surgery.

\section{Clinical Case}

A 41-year-old Japanese woman, gravida 1 para 1, visited to the Department of surgery of our hospital complaining of umbilical nodule for 6 months. The nodule had slowly increased in size and she noticed abdominal pain during the menstrual period. Her medical history was unremarkable without slight dysmenorrhea. She did not take any oral contraceptives and had regular menstrual cycles. Physical examination revealed a brown nodule of approximate $1 \mathrm{~cm}$ in diameter located deep in the umbilical fold. An ultrasound confirmed the presence of a hypo-echonic mass of $10 \mathrm{~mm}$ in the umbilicus. A reconstructed sagittal CT examination showed an umbilical nodular mass (Figure 1). Under the working diagnosis of the umbilical subcutaneous tumor, the tumor was excised with saving the navel, under local anesthesia with Xylocine (lidocaine $\mathrm{HCl}$, Astrazeneca, Osaka, Japan). The lesion was entirely excised deep to the fascia. There was no evidence of connection the peritoneal cavity. The tumor was measured approximate $1.0 \mathrm{~cm}$ in diameter (Figure 2). The tumor was cut in longitudinally and the cut-surface of the tumor was whitish grey. The cut specimens were fixed in $10 \%$ buffered formalin (Nacalai Tesque Co. Ltd., Kyoto, Japan) for 3 days and embedded in paraffin (Wako pure chemical, Osaka, Japan). Four $\mu \mathrm{m}$-thick sections were made and stained with hematoxylin and eosin (Muto pure chemical Co. ltd, Tokyo, Japan) for histoipathological examination. On microscopic examination, glands with stromal cells were found. To determine the presence of endometrial stroma cells, CD10 immunohistochemistry was performed using an antibody against CD10 (Novacastra, Leica Biosystem, Newcastle, UK). The stromal cells expressed cytoplasmic positivity for CD10 (Figure 3). Thus, our pathological diagnosis was made as primary UE. The patient was informed the risk of recurrence at the time of leaving hospital.

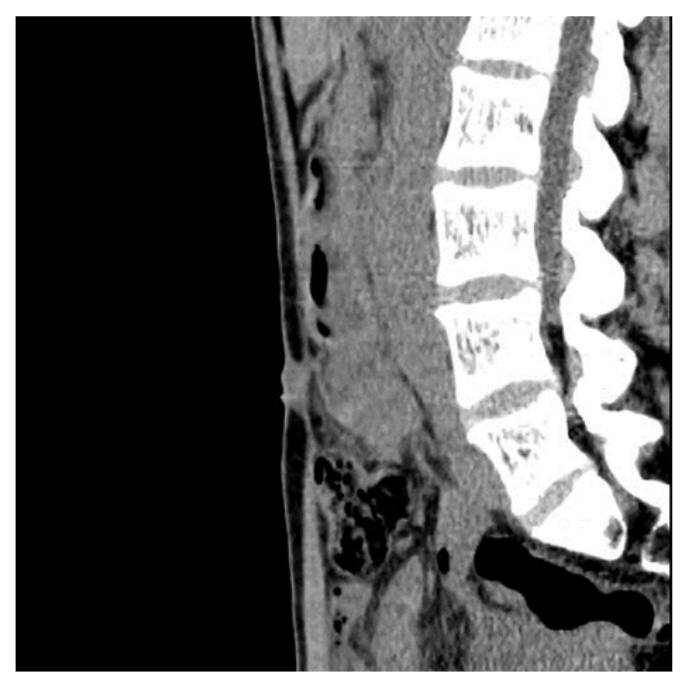

Figure 1. A reconstructed sagittal CT examination shows an umbilical mass. 


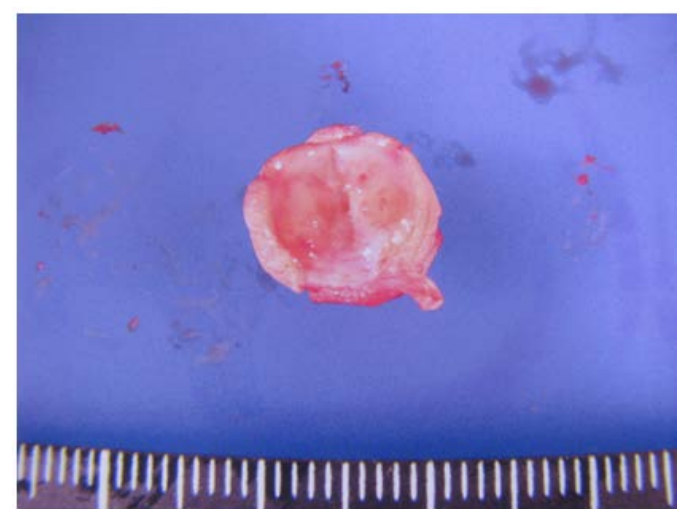

Figure 2. The cut-surface of the tumor $10 \mathrm{~mm}$ in diameter is whitish grey.

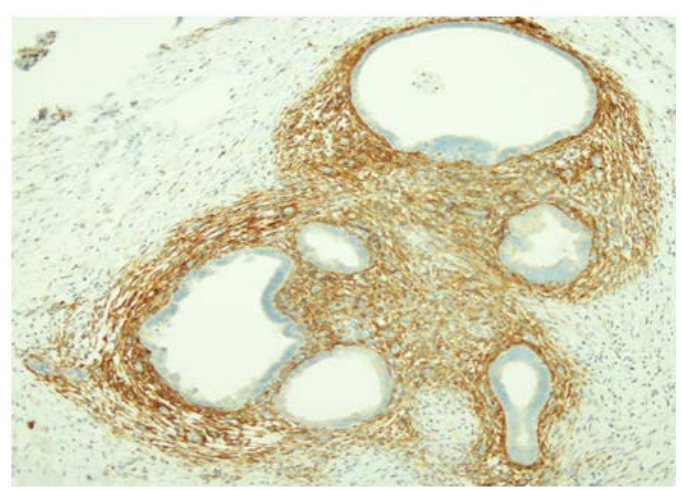

Figure 3. CD10 immunohistochemistry showed positive reaction in the cytoplasm of stromal cells (original magnification $\times 40$ ).

Although postoperative recovery was uneventful, she had noticed slight dysmenorrheal two weeks after the umbilical surgery. Based on the findings of CT and ultrasonography, we suspected endometriotic cyst and pelvic endometriosis. Two months later the first umbilical surgery, she underwent left salpingo-oophorectomy and release of adhesion around the left adnexa under a laparoscope. During the laparoscopic surgery, the endometriotic findings could not be observed in the peritoneum around the back side of umbilicus. The pathological diagnosis was made as ovarian cyst and pelvic peritoneal endometriosis. The second postoperative recovery was also uneventful. She was treated with dienogest ( $2 \mathrm{mg} /$ day) for six months after four injections of GnRH analogue (Leuprorelin acetate, $1.88 \mathrm{mg}$ ) for four months. After three years of the follow-up, there were no signs of local relapses and no other clinical and ultrasonographic abnormalities suggesting endometriosis.

\section{Discussion}

UE has been reported to be $0.4 \%$ - $4 \%$ of all patients with endometriosis and accounts for $30 \%-40 \%$ cases of cutaneous endometriosis [4]. The occurrence of secondary UE can be explained by iatrogenic dissemination of endometrial cells during either laparoscopic or surgical procedures [4]. It is difficult to clarify the origin of primary UE. Different hypotheses have been proposed, such as the embryonic rest theory of Wollfian or Müllerian remnants, the transplantation theory in which the ectopic endometrial tissue harbors from retrograde menstruation or hematogenous/lymphatic dissemination, or a combination of them [3]-[5]. In the present case, endometrial tissues were not observed in the peritoneum around the back side of umbilicus during the laparoscopic surgery. Thus, the embryonic rest theory or hematogenous/lymphatic dissemination is attractive for our case.

Patients with UE are commonly in the reproductive age. Victory et al. reported that the mean age was 37.7 years, with the youngest being 23 years [3]. The typical symptoms are cyclical swelling, pain and bleeding from the umbilicus concomitant with menstrual bleeding [3] [11]. There were a few UE cases without umbilical 
bleeding, as in our case [9] [10].

Surgical excision of UE is recommended with wide and healthy margin [6] in order to make accurate histopathological diagnosis and exclusion of malignancy [12] [13]. The size of UE was reported to be $2.99 \pm 0.20 \mathrm{~cm}$ $(0.5$ to $3 \mathrm{~cm})$ [4]. Since the size $(1 \mathrm{~cm})$ of our UE was small, total umbilical excision was not be necessary [6] and we could preserve her navel without recurrence for three years.

Definitive treatment of UE is surgical excision and subsequent gynecological evaluation for pelvic endometriosis is recommended for all UE patients. Approximate 15\% of UE patients have co-existence with pelvic endometriosis [14]. In this case, our diagnosis and treatment of UE could find symptomatic pelvic endometriosis.

When UE is coexistent with pelvic endometriosis, progesterone and GnRH analogue is useful for treatment of the symptoms and recurrence [2]-[4]. For the prevention of recurrence, the present patient was treated with dienogest and GnRH analogue after the surgical treatment. In case of local excision, UE patients should also be fully informed about the risk of recurrence [3].

High historical risk factors of UE are reported to be previous surgery and time to presentation [4]. To avoid iatrogenic seeding to the umbilicus, a careful endometriotic excision and a laparoscopic trocar with use of a specimen bag are considered to be important [15]. There is a reported case of an endometriosis with malignant transformation in 73-year-old patient with a history of umbilical bleeding from the age of 30 until menopause [16]. No treatment for a long time might lead malignant transformed. Early surgical treatment also may induce narrow excision.

\section{Conclusion}

Although primary UE is extremely rare, UE should be suspected in case of umbilical nodule even in the absence of pelvic endometriosis or previous abdominal surgery. Definitive diagnosis of UE is made by histopathology with immunohistochemistry of CD-10. Since early diagnosis of UE can preserve the navel, surgery is the main treatment for UE. Patients should be counselled about the risk of local recurrence.

\section{Acknowledgements}

We thank the editor and reviewers for their constructive comments, which helped us to improve the manuscript. This case report was approved by the patient verbal consent.

\section{Competing Interests}

The authors declare that they have no competing interests.

\section{References}

[1] Spaziani, E., Picchio, M., Di Filippo, A., De Cristofano, C., Ceci, F. and Stagnitti, F. (2009) Spontaneous Umbilical Endometriosis: A Case Report with One-Year Follow-Up. Clinical Experimental Obstetrics Gynecology, 36, 263-264.

[2] Rosina, P., Pugliarello, S., Colato, C. and Girolomoni, G. (2008) Endometriosis of Umbilical Cicatrix: Case Report and Review of the Literature. Acta Dermatovenerologica Croatica, 16, 218-221.

[3] Bagade, P.V. and Guirguis, M.M. (2009) Menstruating from the Umbilicus as a Rare Case of Primary Umbilical Endometriosis: A Case Report. Journal of Medical Case Reports, 3, 9326. http://dx.doi.org/10.1186/1752-1947-3-9326

[4] Victory, R., Diamond, M.P. and Johns, D.A.J. (2007) Villar's Nodule: A Case Report and Systematic Literature Review of Endometriosis Externa of the Umbilicus. Journal of Minimally Invasive Gynecology, 14, 23-32. http://dx.doi.org/10.1016/j.jmig.2006.07.014

[5] Kyamidis, K., Lora, V. and Kanitakis, J. (2011) Spontaneous Cutaneous Umbilical Endometriosis: Report of A New Case with Immunohistochemical Study and Literature Review. Dermatology Online Journal, 17, 5.

[6] Fancellu, A., Pinna, A., Manca, A., Capobianco, G. and Porcu, A. (2013) Primary Umbilical Endometriosis. Case Report and Discussion on Management Options. International Journal of Surgery Case Reports, 4, 1145-1148. http://dx.doi.org/10.1016/j.ijscr.2013.11.001

[7] Goldberg, J.M. and Bedaiwy, M.A. (2007) Recurrent Umbilical Endometriosis after Laparoscopic Treatment of Minimal Pelvic Endometriosis: a case report. The Journal of Reproductive Medicine, 52, 551-552.

[8] Michowitz, M., Baratz, M. and Stavorovsky, M. (1983) Endometriosis of the Umbilicus. Dermatologica, 167, 326-330. http://dx.doi.org/10.1159/000249810 
[9] Chatzikokkinou, P., Thorfinn, J., Angelidis, I.K., Papa, G. and Trevisan, G. (2009) Spontaneous Endometriosis in an Umbilical Skin Lesion Acta dermatovenerologica Alpina, Panonica, et Adriatica, 18, 126-130.

[10] Dessy, L.A., Buccheri, E.M., Chiummariello, S., Gagliardi, D.N. and Onesti, M.G. (2008) Umbilical Endometriosis, Our Experience. In Vivo, 22, 811-815.

[11] Latcher, J.W. (1953) Endometriosis of the Umbilicus. American Journal of Obstetrics Gynecology, 66, 161-168.

[12] Khaled, A., Hammami, H., Fazaa, B., Zermani, R., Ben Jilani, S. and Kamoun, M.R. (2008) Primary Umbilical Endometriosis: A Rare Variant of Extragenital Endometriosis. Pathologica, 100, 473-475.

[13] Obata, K., Ikoma, N., Oomura, G. and Inoue, Y. (2013) Clear Cell Adenocarcinoma Arising from Umbilical Endometriosis. Journal of Obstetrics and Gynaecology Research, 39, 455-461. http://dx.doi.org/10.1111/j.1447-0756.2012.01964.x

[14] Dadhwal, V., Gupta, B., Dasgupta, C., Shende, U. and Deka, D. (2011) Primary Umbilical Endometriosis: A Rare Entity. Archives of Gynecology and Obstetrics, 283, 119-120. http://dx.doi.org/10.1007/s00404-010-1809-2

[15] Lauslahti, K. (1972) Malignant External Endometriosis: A Case of Adenocarcinoma of Umbilical Endometriosis. Acta Pathologica et Microbiologica Scandinavica, Section A, Pathology, 233, 98-102.

[16] Barbaros, U., Iyibozkurt, A.C., Gulluoglu, M., Barbaros, M., Erbil, Y., Tunali, V. and Mercan, S. (2005) Endometriotic Umbilical Port Site Metastasis after Laparoscopy. American Journal of Obstetrics Gynecology, 193, 1761-1763. http://dx.doi.org/10.1016/j.ajog.2005.05.088 
Scientific Research Publishing (SCIRP) is one of the largest Open Access journal publishers. It is currently publishing more than 200 open access, online, peer-reviewed journals covering a wide range of academic disciplines. SCIRP serves the worldwide academic communities and contributes to the progress and application of science with its publication.

Other selected journals from SCIRP are listed as below. Submit your manuscript to us via either submit@scirp.org or Online Submission Portal.
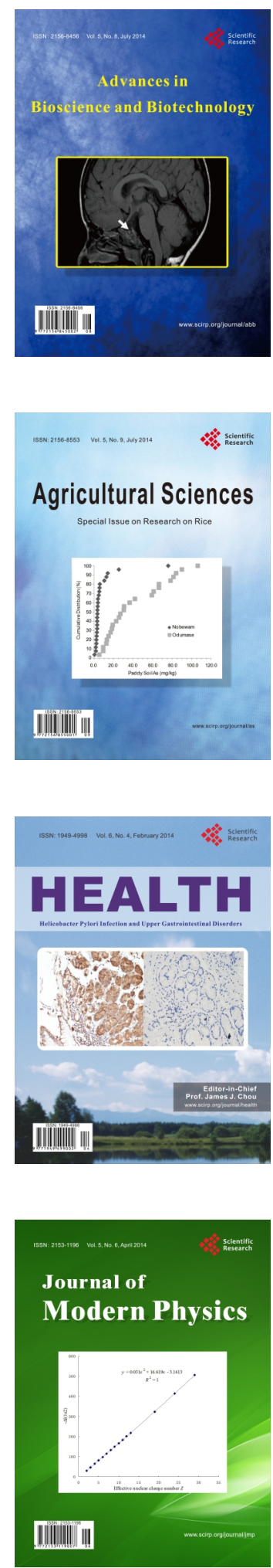
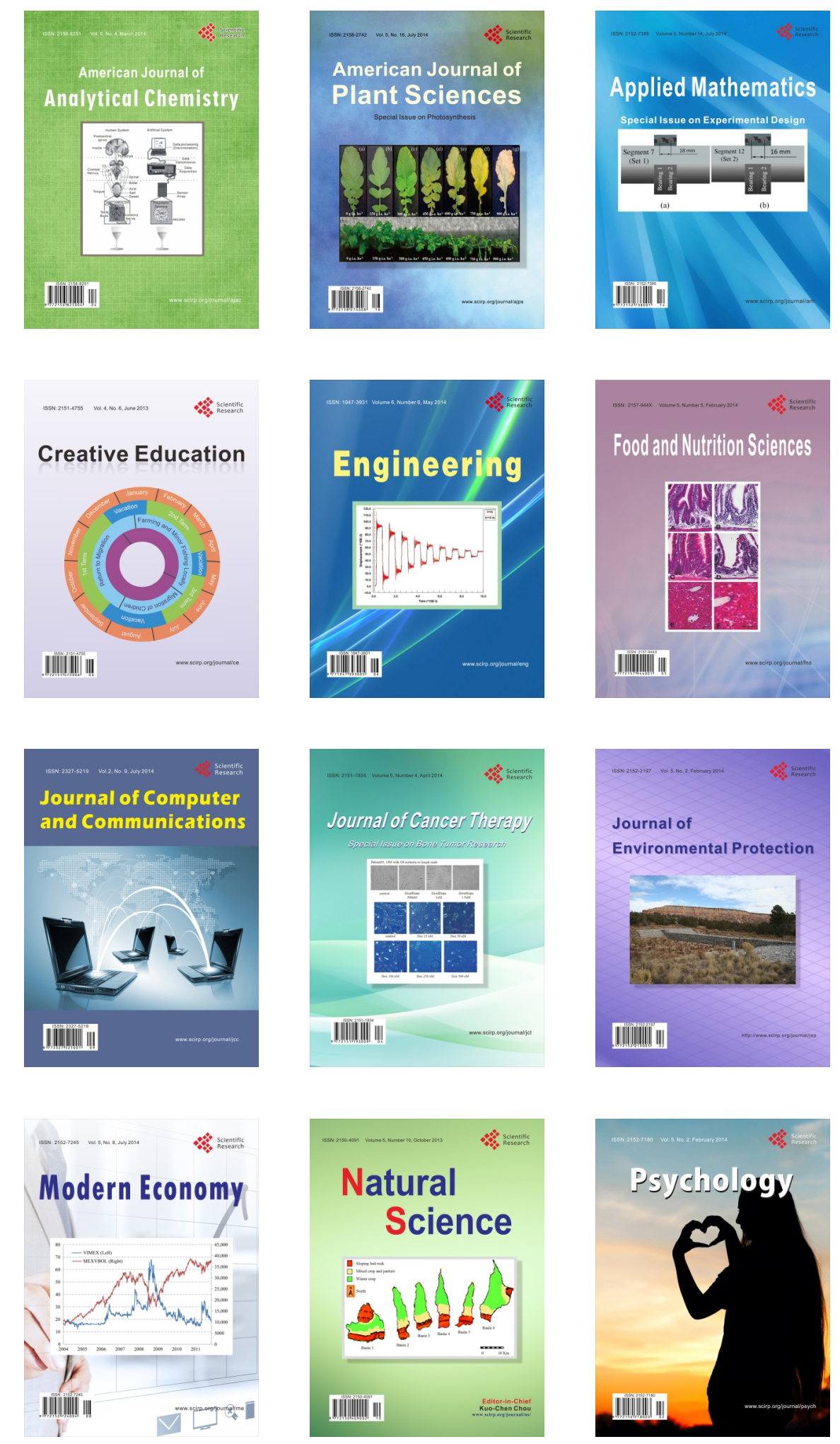